\title{
Extração do óleo da polpa do abacate com Metanol e Etanol a diferentes temperaturas
}

\author{
Oil extraction from the avocado pulp with methanol and ethanol at different \\ temperatures
}

\author{
Alessandro Cazonatto Galvão, Milena Copetti, Camilla Dal Magro, \\ Sidiane Iltchenco, Weber da Silva Robazza \\ Departamento de Engenharia de Alimentos, Universidade do Estado de Santa Catarina - UDESC, \\ Pinhalzinho - Santa Catarina - Brasil \\ alessandro.galvao@udesc.br
}

\begin{abstract}
Resumo
O objetivo do presente trabalho consistiu na extração do óleo da polpa do abacate de variedade Hass utilizando como solvente o etanol e o metanol. Os experimentos foram conduzidos a pressão atmosférica e no intervalo de temperatura entre 283,15 Ke 313,15 K. As extrações foram realizadas por um período de 10 horas com amostragens a diferentes tempos de extração. Para ambos os solventes a capacidade de extração apresentou resultados crescentes com o aumento da temperatura. O solvente etanol mostrou maior capacidade de solubilização do óleo da polpa do abacate. Os resultados experimentais foram correlacionados a um modelo que considera que o processo de extração ocorre por dois fenômenos; primeiro o óleo que está na superfície do sólido é rapidamente solubilizado pelo solvente e segundo o óleo residual é removido do interior do sólido por processo difusional.
\end{abstract}

Palavras-chave: abacate, extração, óleo, modelagem

\begin{abstract}
The purpose of this work was to perform the oil extraction from the pulp of the variety Hass avocado by using the solvents methanol and ethanol in the temperature range between $283.15 \mathrm{~K}$ and $313.15 \mathrm{~K}$ and under atmospheric pressure. The extraction was conducted for a period of 10 hours with sampling at different extraction times. For both solvents it was observed an increase of oil extraction with the increase of temperature. Ethanol was the solvent which showed higher capacity to solubilize the oil. The experimental data were fitted to a model that considers the extraction based on two simultaneous processes. First, the oil present on the seed surface is quickly solubilesed by the solvent and second, the extraction of the remaining oil present inside the cells is governed by a diffusion process.
\end{abstract}

Keywords: avocado, extraction, oil, modeling 


\section{Introdução}

O abacate é uma fruta tropical, sendo cultivado por quase todo o mundo, pertencendo ao gênero Persea americana e a família das Lauraceae (KHAN e ABOURASHED, 1980). No Brasil, o consumo do abacate é feito in natura ou na forma de vitaminas, sorvetes e sobremesas. Dentre os componentes presentes no abacate, destacam-se a elevada quantidade de ácidos graxos, proteínas e vitaminas lipossolúveis (ORTIZ et al., 2004).

A variedade Hass destaca-se das demais variedades de abacate, devido ao sabor, cor, sazonalidade, por ser menos perecível e também pelo alto conteúdo de óleo (KURLAENDER, 2005). Por apresentar elevado teor de lipídios em sua composição, a variedade Hass pode ser considerada uma alternativa promissora na produção de óleo, com potencial comercial a ser explorado (MOOZ et al., 2012).

Frutos como o abacate, que apresentam altos teores de lipídeos na polpa podem ser uma matéria -prima importante para a obtenção de óleo (REQUEJO, 1999). Devido às alterações sofridas pelo fruto durante o processo de maturação, pouco óleo é encontrado na polpa do abacate ainda verde, apresentando maiores concentrações de óleo quando o fruto se encontra maduro (MOSTERT et al., 2007).

O óleo de abacate pode ser utilizado nas indústrias farmacêutica, cosmética e alimentícia (KURLAENDER, 2005; SWISHER, 1988). Como a composição dos ácidos graxos do óleo de abacate é semelhante à composição dos ácidos graxos do óleo de oliva, é possível a substituição do óleo de oliva pelo óleo de abacate, ou então, a possibilidade de mesclar os dois óleos, levando a uma diminuição da importação do azeite de oliva no Brasil e incorporando maior valor ao produto nacional (SOARES et al., 1992). A composição apresenta uma quantidade apreciável das vitaminas lipossolúveis A, D e E (REQUEJO, 1999) além de ácido oleico (63,73\%), linolênico $(15,27 \%)$, palmítico $(14,80 \%)$, palmitoleico $(4,86 \%)$ e linoleico (1,09\%) (YANTY et al., 2011).

Muitos métodos para a extração do óleo da polpa do abacate vem sendo estudados, tais como: extração por centrifugação; extração por solvente; extração assistida por micro-ondas; extração por prensagem hidráulica contínua ou descontínua (BERASATEGI et al., 2012; MORENO et al., 2003; BIZIMANA et al., 1993).

O método de extração por solvente a partir de um abacate desidratado extrai quase todos os óleos presentes (KURLAENDER, 2005). Um solvente convencional utilizado nos processos extrativos é o hexano, porém este solvente apresenta elevada toxicidade, provém de uma fonte não renovável de energia e é altamente inflamável. Os solventes etanol e metanol tem apresentado um rendimento alto na extração de óleos vegetais, além de serem oriundos de fontes renováveis de energia e apresentarem menor grau de toxicidade (LAMB e ADIN, 1983; HOTZA e DINIZ DA COSTA, 2008; ISSARIYAKUL et al., 2007; CUEVAS et al., 2009).

A busca por um planeta sustentável tem motivado os grupos de pesquisa a desenvolverem novos processos de extração, empregando solventes renováveis. Diante disto, mesmo que a toxicidade do metanol deva ser considerada, alcoóis de cadeia curta como metanol e etanol parecem ser respostas para algumas demandas visto que ambos podem ser produzidos por rota fermentativa.

O presente trabalho teve por objetivo estudar a extração do óleo da polpa de abacate com metanol e etanol nas temperaturas de 283,15 K, 293,15 K, 303,15 K e 313,15 K sob pressão atmosférica e por um período de tempo de até 10 horas.

\section{Materiais e métodos}

Para este estudo, foram adquiridos abacates da variedade Hass, obtidos no comércio da cidade de Chapecó (SC). Os abacates se apresentavam no estágio final de maturação e foram despolpados com o auxílio de uma colher. Logo após, a polpa foi amassada e homogeneizada com um garfo. Os solventes utilizados neste trabalho, metanol (Biotec - Brasil - 99,8\%) e etanol (Biotec - Brasil - 99,9\%), não receberam nenhum tratamento adicional.

A umidade da polpa foi determinada mantendo-se aproximadamente 5,0 gramas da polpa homogeneizada em estufa a $353,15 \mathrm{~K}$ até peso constante. $\mathrm{O}$ restante do material foi seco em estufa a 353,15 K por um período superior a 96 horas e subsequentemente moído utilizando um liquidificador. Na sequência, o material foi fracionado, acondicionado em sacos plásticos e mantidos em temperatura aproximada de 268,15 K até a condução do experimento.

A extração do óleo realizou-se em um vaso de vidro encamisado com procedimento conduzido em batelada agitada magneticamente e com temperatura controlada por um banho termostático. As extrações foram efetuadas nas temperaturas de 283,15 K, 293,15 K, 303,15 K e 313,15 K. A extração foi feita utilizando uma relação mássica de solvente para sólido de 20:1, visando uma maior extração de óleo (IVERSON et al., 2001; NEVES et al., 2001). Durante a extração foram coletadas amostras de aproximadamente $10 \mathrm{ml} \mathrm{em}$ tempos pré-determinados e o teor de óleo nestas amostras foram determinados. 
As amostras da micela (óleo extraído + solvente), removidas do vaso de extração, foram transferidas para um balão de fundo chato de $250 \mathrm{ml}$ e tiveram sua massa determinada. Logo após, o balão contendo a micela foi submetido à separação do solvente em um rota-evaporador. Em seguida o balão foi transferido para estufa a 353,15 K para a remoção residual de solvente por um período de 24 horas. Após a remoção do solvente o balão contendo óleo foi resfriado em dessecador e a concentração de óleo determinada.

\section{Resultados e discussões}

A umidade da polpa do abacate selecionado para este estudo foi de $66,18 \%$. O resultado encontrado foi semelhante ao apresentado na literatura (BIZIMANA et al., 1993; ORTIZ et al., 2004). É importante colocar que valores de umidade para uma mesma espécie de abacate podem variar de acordo com o estágio de maturação, localização geográfica da planta e época adequada para colheita (GAYDOU et al., 1987; OZDEMIR e TOPUZ, 2004).

Os resultados de concentração de óleo foram determinados pela Equação (1) em que $M_{b o}$ representa a massa de óleo mais a massa do balão, $M_{b}$ a massa do balão, $M_{S}$ a massa de solvente presente na amostra, $M_{S S}$ a massa de sólido seco submetido a extração e $S$ a massa de solvente utilizado na extração.

$$
\text { [óleo] }=\frac{M_{b o}-M_{b}}{M_{s}} \times \frac{S}{M_{s s}}
$$

Na Tabela 1 são apresentados os resultados encontrados para a concentração de óleo, em base seca, em função do tempo e da temperatura de extração para os solventes estudados.

As incertezas experimentais apresentadas na Tabela 1 foram determinadas aplicando-se o método de propagação de incertezas (PUGH e WINSLOW, 1966), baseado na Equação (2).

$F=F(x, y, \ldots) \rightarrow \delta(F)=\left(\left(\frac{\partial F}{\partial x}\right)^{2} \cdot(\delta x)^{2}+\left(\frac{\partial F}{\partial y}\right)^{2} \cdot(\delta y)^{2}+\cdots\right)^{\frac{1}{2}}$

O comportamento das extrações em função da temperatura e do tempo para os solventes investigados é apresentado nas Figuras 1 e 2.

Analisando a Tabela 1, é observado que tanto na extração com metanol, como na extração com etanol, existe uma dependência crescente da extração com a temperatura. Este comportamento pode ser explicado por um aumento da difusão do solvente na estrutura do sólido devido a uma diminuição da viscosidade do solvente com um aumento da temperatura (TREYBAL, 1977).

Tabela 1: Tabela 1: Concentração de óleo em função do tempo e da temperatura de extração para os solventes estudados

\begin{tabular}{|c|c|c|c|c|}
\hline \multirow{2}{*}{$\begin{array}{c}\mathrm{T} \\
(\mathrm{K})\end{array}$} & \multirow[t]{2}{*}{$t(\min )$} & $\mathrm{CH}_{3} \mathrm{OH}$ & \multirow{2}{*}{$\mathrm{t}(\mathrm{min})$} & \multirow{2}{*}{ [óleo] $\left(\mathrm{g}_{\text {óleo }} \cdot \mathrm{g}^{-1}\right.$ sólido seco } \\
\hline & & [óleo] $\left(\mathrm{g}_{\text {óleo }} \cdot \mathrm{g}^{-1}\right.$ sólido seco $)$ & & \\
\hline \multirow[t]{7}{*}{283,15} & 20 & $0,0308 \pm 0,0005$ & 20 & $0,1301 \pm 0,0003$ \\
\hline & 40 & $0,0672 \pm 0,0003$ & 40 & $0,2267 \pm 0,0003$ \\
\hline & 60 & $0,0892 \pm 0,0003$ & 60 & $0,2807 \pm 0,0003$ \\
\hline & 120 & $0,0946 \pm 0,0002$ & 120 & $0,3312 \pm 0,0004$ \\
\hline & 240 & $0,0971 \pm 0,0002$ & 240 & $0,3556 \pm 0,0002$ \\
\hline & 420 & $0,0984 \pm 0,0003$ & 420 & $0,3669 \pm 0,0004$ \\
\hline & 600 & $0,0992 \pm 0,0003$ & 600 & $0,3683 \pm 0,0004$ \\
\hline \multirow[t]{7}{*}{293,15} & 20 & $0,0505 \pm 0,0003$ & 20 & $0,1994 \pm 0,0004$ \\
\hline & 40 & $0,0930 \pm 0,0002$ & 40 & $0,3248 \pm 0,0003$ \\
\hline & 60 & $0,1107 \pm 0,0002$ & 60 & $0,3887 \pm 0,0002$ \\
\hline & 120 & $0,1180 \pm 0,0003$ & 120 & $0,4232 \pm 0,0002$ \\
\hline & 240 & $0,1192 \pm 0,0003$ & 240 & $0,4375 \pm 0,0002$ \\
\hline & 420 & $0,1212 \pm 0,0003$ & 420 & $0,4461 \pm 0,0002$ \\
\hline & 600 & $0,1229 \pm 0,0003$ & 600 & $0,4487 \pm 0,0002$ \\
\hline \multirow[t]{7}{*}{303,15} & 20 & $0,0769 \pm 0,0002$ & 20 & $0,3201 \pm 0,0005$ \\
\hline & 40 & $0,1108 \pm 0,0003$ & 40 & $0,4294 \pm 0,0004$ \\
\hline & 60 & $0,1230 \pm 0,0003$ & 60 & $0,4592 \pm 0,0003$ \\
\hline & 120 & $0,1284 \pm 0,0003$ & 120 & $0,4886 \pm 0,0005$ \\
\hline & 240 & $0,1314 \pm 0,0002$ & 240 & $0,5132 \pm 0,0002$ \\
\hline & 420 & $0,1337 \pm 0,0002$ & 420 & $0,5215 \pm 0,0002$ \\
\hline & 600 & $0,1349 \pm 0,0004$ & 600 & $0,5354 \pm 0,0002$ \\
\hline \multirow[t]{7}{*}{313,15} & 20 & $0,1001 \pm 0,0002$ & 20 & $0,3875 \pm 0,0002$ \\
\hline & 40 & $0,1287 \pm 0,0002$ & 40 & $0,5403 \pm 0,0003$ \\
\hline & 60 & $0,1371 \pm 0,0003$ & 60 & $0,5904 \pm 0,0003$ \\
\hline & 120 & $0,1434 \pm 0,0003$ & 120 & $0,6213 \pm 0,0003$ \\
\hline & 240 & $0,1457 \pm 0,0003$ & 240 & $0,6381 \pm 0,0004$ \\
\hline & 420 & $0,1468 \pm 0,0002$ & 420 & $0,6527 \pm 0,0002$ \\
\hline & 600 & $0,1474 \pm 0,0003$ & 600 & $0,6654 \pm 0,0002$ \\
\hline
\end{tabular}

Em relação à taxa de extração, é observado um aumento na concentração do óleo extraído até aproximadamente 240 minutos. Após este período, observa-se que há uma tendência a estabilização da curva de extração, indicando que a extração se aproxima da condição de equilíbrio. Este comportamento foi constatado tanto para a extração com etanol como para a extração com metanol podendo o comportamento ser analisado nas Figuras 1 e 2. 


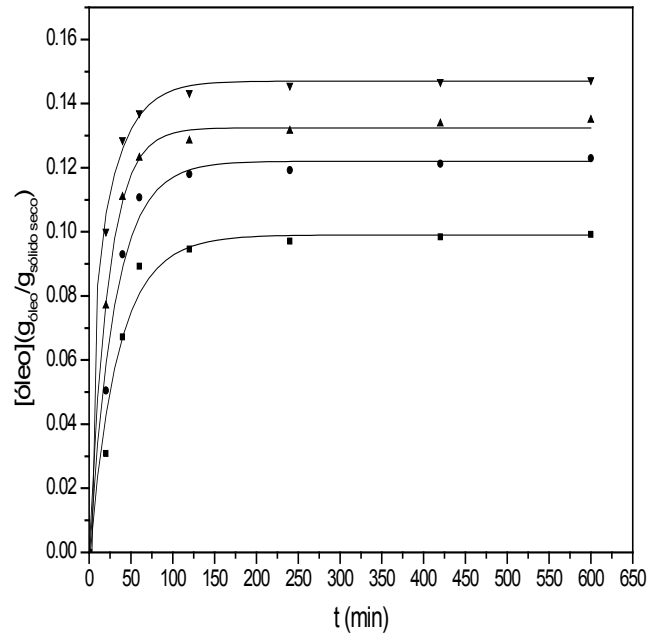

Figura 1: Comportamento da extração do óleo com metanol em função do tempo e da temperatura de extração: $\mathbf{~ 2 8 3 , 1 5 ~}$ K; • 293,15 K; $\boldsymbol{\Delta} 303,15 \mathrm{~K} ; \boldsymbol{\nabla} 313,15 \mathrm{~K}$

Avaliando os resultados dispostos na Tabela $1 \mathrm{e}$ nas Figuras 1 e 2 constata-se que o etanol foi o solvente com maior capacidade de extração do óleo da polpa do abacate. Este comportamento pode ser explicado devido a polaridade das moléculas envolvidas na extração. $\mathrm{O}$ solvente etanol com constante dielétrica de 24,5 é menos polar que o metanol com constante dielétrica de 32,7. Em contrapartida, lipídeos costumam ser fracamente polares apresentando constante dielétrica entre 2 e 4 (DAMODARAN et al., 2007). Dessa forma, o solvente menos polar é mais soluvel nos óleos vegetais levando a maiores taxas de solubilização e consequentemente maiores capacidades de extração.

As linhas contínuas que correlacionam os dados experimentais apresentados nas Figuras 1 e 2 representam o comportamento do modelo utilizado (SO e MACDONALD, 1986).

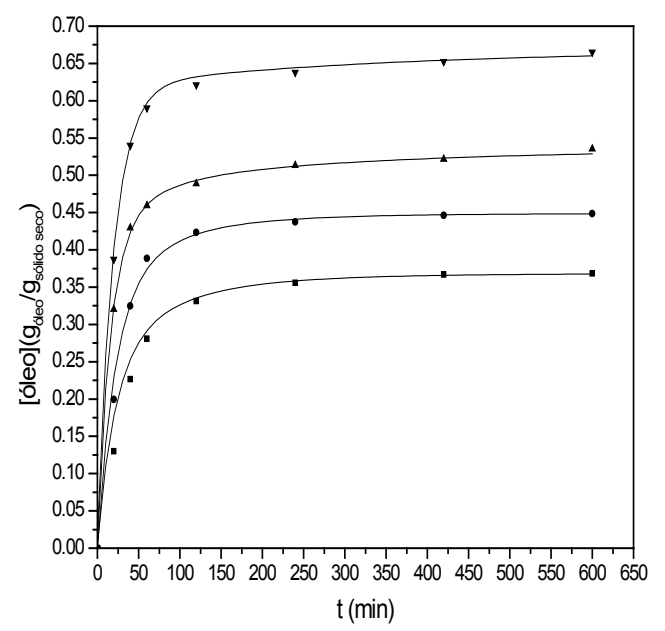

Figura 2: Comportamento da extração do óleo com etanol em

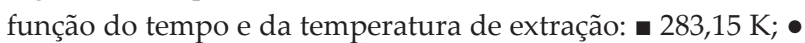
293,15 K; $\Delta$ 303,15 K; $\boldsymbol{\nabla} 313,15 \mathrm{~K}$
O modelo considera que o processo de extração ocorre por dois fenômenos que acontecem simultaneamente. Primeiro o óleo que está na superfície do sólido é rapidamente solubilizado (lavado) pelo solvente no início do processo de extração. O segundo fenômeno trata da remoção do óleo residual do interior do sólido por processo difusional. O processo difusional ocorre com duas contribuições; a primeira, mais rápida, trata da remoção do óleo do interior de células que se encontram rompidas e a segunda, lenta, que trata da remoção do óleo do interior de células intactas. O equacionamento do modelo, expresso na forma de concentração ( $g_{\text {óleo }} / 100 g_{\text {solvente }}$ ) é expresso pela Equação (3) em que $C_{t}$ representa a concentração de óleo em um tempo $t, C_{e}^{w}$ e $C_{e}^{d}$ são as concentrações de equilíbrio no processo de lavagem e no processo difusional respectivamente. Os coeficientes de transferência de massa são $k_{w}$ e $k_{d}$ e os indices 1 e 2 representam o primeiro e o segundo processo de difusão. A Equação (4) representa a concentração de equilíbrio para um tempo muito grande $(t \rightarrow \infty)$.

$C_{t}=C_{e}^{w}\left(1-\mathrm{e}^{-k_{w} t}\right)+C_{e}^{d 1}\left(1-\mathrm{e}^{-k_{d 1} t}\right)+C_{e}^{d 2}\left(1-\mathrm{e}^{-k_{d 2} t}\right)$

$C_{e}=C_{e}^{w}+C_{e}^{d 1}+C_{e}^{d 2}$

A fim de modelar os dados expressos em góleo $/$ $\mathrm{g}_{\mathrm{ss}}$ as equações do modelo foram utilizadas na forma como apresentado nas Equações (5) e (6) (MEZIANE e KADI, 2008).

$\rho_{t}=\rho_{e}-\rho_{e}^{w}\left(\mathrm{e}^{-k_{w} t}\right)-\rho_{e}^{d 1}\left(\mathrm{e}^{-k_{d 1} t}\right)-\rho_{e}^{d 2}\left(\mathrm{e}^{-k_{d 2} t}\right)$

$\rho_{e}=\rho_{e}^{w}+\rho_{e}^{d 1}+\rho_{e}^{d 2}$

Analisando o comportamento do modelo apresentado nas Figuras 1 e 2 juntamente com os valores do coeficiente de ajuste apresentados na Tabela 2, observa-se que o modelo utilizado é capaz de correlacionar satisfatoriamente os resultados experimentais.

Os coeficientes de transferência de massa determinados pela modelagem indicam que o estágio de lavagem tem predominância sobre os estágios difusionais e que o primeiro estágio de difusão tem predominância sobre o segundo. Observa-se também um aumento dos coeficientes de transferência de massa com o aumento da temperatura.

Na Tabela 2 são apresentados os valores para os parâmetros ajustáveis do modelo e os coeficientes do ajuste. 
Tabela 2: Efeito da temperatura nos coeficientes de transferência de massa e nas concentrações de equilíbrio

\begin{tabular}{|c|c|c|c|c|c|c|c|}
\hline $\mathrm{T}(\mathrm{K})$ & $\begin{array}{c}\boldsymbol{k}_{w} \\
\left(\mathrm{~min}^{-1}\right)\end{array}$ & $\begin{array}{c}\boldsymbol{k}_{d 1} \\
\left(\min ^{-1}\right)\end{array}$ & $\begin{array}{c}\boldsymbol{k}_{d 2} \\
\left(\min ^{-1}\right)\end{array}$ & $\begin{array}{c}\rho_{e}^{w} \\
\left(\frac{\mathbf{g}_{0 \text { leo }}}{\mathbf{g}_{s s}}\right)\end{array}$ & $\begin{array}{c}\rho_{e}^{d 1} \\
\left(\frac{\mathbf{g}_{\text {olleo }}}{\mathrm{g}_{\mathrm{ss}}}\right)\end{array}$ & $\begin{array}{c}\rho_{e}^{d 2} \\
\left(\frac{g_{\text {olleo }}}{\mathrm{g}_{s s}}\right)\end{array}$ & $R^{2}$ \\
\hline \multicolumn{8}{|c|}{$\mathrm{CH}_{3} \mathrm{OH}$} \\
\hline 283,15 & 0,0541 & 0,0327 & 0,0062 & 0,0672 & 0,0246 & 0,0073 & 0,9614 \\
\hline 293,15 & 0,0607 & 0,0419 & 0,0118 & 0,0814 & 0,0301 & 0,0115 & 0,9815 \\
\hline 303,15 & 0,0803 & 0,0524 & 0,0209 & 0,0977 & 0,0322 & 0,0049 & 0,9912 \\
\hline 313,15 & 0,1038 & 0,0626 & 0,0382 & 0,1123 & 0,0319 & 0,0032 & 0,9933 \\
\hline \multicolumn{8}{|c|}{$\mathrm{CH}_{3} \mathrm{CH}_{2} \mathrm{OH}$} \\
\hline 283,15 & 0,0372 & 0,0124 & 0,0023 & 0,2132 & 0,0820 & 0,0730 & 0,9751 \\
\hline 293,15 & 0,0507 & 0,0172 & 0,0034 & 0,3046 & 0,0998 & 0,0442 & 0,9935 \\
\hline 303,15 & 0,0653 & 0,0187 & 0,0053 & 0,4119 & 0,1024 & 0,0212 & 0,9994 \\
\hline 313,15 & 0,0712 & 0,0463 & 0,0081 & 0,5218 & 0,1379 & 0,0084 & 0,9979 \\
\hline
\end{tabular}

A dependência dos coeficientes de transferência de massa com a temperatura pode ser descritas pela equação de Arrhenius representada pela Equação (7) em que A é o fator de frequência, $E_{a}$ é a energia de ativação e R é a constante universal dos gases.

$$
k=A \mathrm{e}^{\frac{-E_{a}}{R T}}
$$

Os parâmetros ajustados a cada uma das etapas descritas pelo modelo juntamente com os coeficientes de ajuste são apresentados na Tabela 3.

Tabela 3: Parâmetros da equação de Arrhenius e coeficiente de ajuste

\begin{tabular}{cccc}
\hline & Lavagem & Difusão 1 & Difusão 2 \\
\hline $\boldsymbol{E}_{\boldsymbol{a}}\left(\mathbf{k J} \cdot \mathbf{~ m o l}^{-\mathbf{1}}\right)$ & 16,4 & $\mathrm{CH}_{3} \mathrm{OH}$ \\
$\boldsymbol{A}\left(\mathbf{m i n}^{-\mathbf{1}}\right)$ & 54,4 & 16,0 & 44,4 \\
$\boldsymbol{R}^{\mathbf{2}}$ & 0,9643 & 29,9 & 965112,5 \\
& & 0,9981 & 0,9995 \\
$\boldsymbol{E}_{\boldsymbol{a}}\left(\mathbf{k J} \cdot \mathbf{m o l}^{-\mathbf{1}}\right)$ & 16,3 & $\mathrm{CH}_{3} \mathrm{CH}_{2} \mathrm{OH}$ & \\
$\boldsymbol{A}\left(\mathbf{m i n}^{-\mathbf{1}}\right)$ & 39,8 & 29,5 & 31,1 \\
$\boldsymbol{R}^{\mathbf{2}}$ & 0,9609 & 0,8358 & 1213,4 \\
\hline
\end{tabular}

Avaliando o desempenho da equação de Arrhenius na correlação dos valores de coeficiente de transferência de massa, observa-se que com exceção da primeira etapa difusional utilizando etanol, todas as demais apresentam fator de correlação superior a 0,95. A energia de ativação para todas as etapas de extração consideradas apresentam valores baixos indicando que o processo de extração é de natureza física.

\section{Conclusão}

A extração do óleo da polpa de abacate pode ser realizada utilizando os solventes etanol e metanol, com maiores concentrações de óleo na extração quando utilizado o etanol. Esse comportamento indica uma dependência da extração em relação ao tamanho da cadeia carbônica do n-alcool.

A performance da extração é função do tempo e da temperatura de extração. Utilizando-se de polpa de abacate seca e moida, 180 minutos de extração são suficientes para uma aproximação da condição de equilíbrio da extração, podendo ser alcançada maiores concentrações com temperaturas de extração mais elevadas.

O modelo utilizado é capaz de correlacionar os dados experimentais utilizando seis parâmetros ajustáveis. Os resultados indicam que no processo de extração utilizado, a etapa de lavagem é predominante sobre as etapas de difusão. O comportamento dos coeficientes de transferência de massa indicam que a extração realizada é governada por processo físico.

\section{Referências bibliográficas}

BERASATEGI, I.; BARRIUSO, B.; ANSONERA, D.; ASTIASARÁN, I. Stability of avocado oil during heating: comparative study to olive oil. Food Chemistry, v. 132, p.439-446, 2012. 
BIZIMANA, V.; BREENE, W.M.; CSALLANY, A.S. Avocado oil extraction with appropriate technology for developing countries. Journal of the American Oil Chemists Society, v. 70, p.821822, 1993.

CUEVAS, M.S.; RODRIGUES, C.E.C.; MEIRELLES, A.J.A. Effect of solvent hydration and temperature in the deacidification process of sunflower oil using ethanol. Journal of Food Engineering, v. 95, p.291-297, 2009.

DAMODARAN, S.; PARKIN, K.; FENNEMA, O.R. Fennema's food chemistry, 4th ed., CRC Press, 2007.

GAYDOU, E.M.; LOZANO, Y.; RATOVOHERY, $\mathrm{J}$. Triglyceride and fatty acid compositions in the mesocarp of Persea americana during fruit development. Phytochemistry, v. 26, p.1595-1597, 1987.

HOTZA, D.; DINIZ DA COSTA, J.C. Fuel cells development and hydrogen production from renewable resources in Brazil. International Journal of Hydrogen Energy, v. 33, p.4915-4935, 2008.

ISSARIYAKUL, T.; KULKARNI, M.G; DALAI, A.K.; BAKHSHI, N.N. Production of biodiesel from waste fryer grease using mixed methanol/ ethanol system. Fuel Processing Technology, v. 88, p.429-436, 2007.

IVERSON, S.J.; LANG, S.L.C.; COOPER, M.H. Comparison of the bligh and dyer and folch methods for total lipid determination in a broad range of marine tissue. Lipids, v. 36, p.1283-1287, 2001.

KHAN, I.A.; ABOURASHED, E.A. Leung's encyclopedia of common natural ingredienta used in food, drugs and cosmetics, 3th ed., Wiley, 1980.

KURLAENDER, A. Avocados. Em: Processing fruits: science and technology. BARRETT, D.M.; SOMOGYI, L.P.; RAMASWAMY, H.S. Boca Raton, CRC Press, 2005.

LAMB, S.I.; ADIN, N.E. Polar organic pollutants in air: a review of collection and analysis. Environment International, v. 9, p.225-243, 1983.
MEZIANE, S.; KADI, H. Kinetics and thermodynamics of oil extraction from olive cake. Journal of the American Oil Chemists Society, v. 85, p.391-396, 2008.

MOOZ, E.D.; GAIANO, N.M; SHIMANO, M.Y.H.; AMANCIO, R.D.; SPOTO, M.H.F. Physical and chemical characterization of the pulp of different varieties of avocado targeting oil extraction potential. Ciência e Tecnologia de Alimentos, v. 32, p.274-280, 2012.

MORENO, A.O.; DORANTES, L.; GALÍNDEZ, J.; GUZMÁN, R.I. Effect of different extraction methods on fatty acids, volatile compounds, and physical and chemical properties of avocado (persea americana mill.) oil. Journal of Agricultural and Food Chemistry, v. 51, p.22162221, 2003.

MOSTERT, M.E.; BOTHA B.M.; PLESSIS, L.M.; DUODU, K.G. Effect of fruit ripeness and method of fruit drying on the extractability of avocado oil with hexane and supercritical carbon dioxide. Journal of the Science of Food and Agriculture, v. 87, p.2880-2885, 2007.

NEVES, V.A.; LOURENÇO, E.J.; SILVA, M.A. Extração, isolamento e fracionamento da proteína de tremoço (Lupinus albus) var. Multolupa. Alimentos e Nutrição, v. 12, p.115-130, 2001.

ORTIZ, M.A.; DORANTES, A.L.; GALÍNDEZ, M.J.; CARDENAS, S.E. Effect of a novel oil extraction method on avocado (persea americana mill) pulp microstructure. Plant Foods for Human Nutrition, v. 59, p.11-14, 2004.

OZDEMIR, F.; TOPUZ, A. Changes in dry matter, oil content and fatty acids composition of avocado during harvesting time and post-harvesting ripening period. Food Chemistry, v. 86, p.79-83, 2004.

PUGH, E.M; WINSLOW, G.H. The analysis of physical measurements, Addison-Wesley, Reading, Mass., 1966.

REQUEJO, C. International trends in fresh avocado and avocado oil production and seasonal variation of fatty acids in new zealand-grown cv. Hass. Dissertation (Masters in Applied Science in Agribusiness Agribusiness) - Massey University New Zealand. Tapia, 1999. 
SOARES, S.E.; MANCINI FILHO, J.; DELLA MODESTA, R.C. Sensory detection limits of avocado oil in mixtures with olive oil. Revista Espanõla de Ciencia y Tecnologia de Alimentos, v. 32, p.509-516, 1992.

SO, G.C.; MACDONALD, D.G. Kinetics of oil extraction from Canola (Rapeseed). The Canadian Journal of Chemical Engineering, v. 64, p80-86, 1986.

SWISHER, H.E. Avocado oil: from food use to skin care. Journal of the American Oil Chemists Society, v. 65, p.1704-1706, 1988.

TREYBAL, R.E. Mass transfer operations. 3th ed., MacGraw-Hill Kogakusha Ltd., 1977.

YANTY, N.A.M.; MARIKKAR, J.M.N.; LONG, K. Effect of varietal differences on composition and thermal characteristics of avocado oil. Journal of the American Oil Chemists Society, v. 88, p.19972003, 2011. 